\title{
Low-Temperature Plasma Modification of Multiwalled Carbon Nanotubes for Advanced Polymer Composites
}

\author{
Siciński M. ${ }^{1}$, Gozdek T. ${ }^{1}$, Bieliński D. M. ${ }^{1}$, Szymanowski H. ${ }^{2}$, and Piątkowska A. ${ }^{3}$
}

\begin{abstract}
In polymer industry there still is a room for new fillers, which can improve composites performance or introduce a new function to the material. The nature of their surface is the main attribute of fillers, as surface energy and specific area determine the compatibility of filler with rubber matrix and the affinity to other c ingredients. One of the major prob-lems is the tendency of fillers to agglomeration - formation of bigger secondary structures, associated with lower level of filler dispersion, what is reflected by the decrease of mechanical properties of rubber vulcanizates (Bielinski at al. ; 2008). Very often it is hard to obtain a well dispersed system for such fillers - it's particles are not wet properly by the polymer during mixing. As a consequence, the total effect of mechanical properties improvement of a composite is lower than expected. Modern fillers like carbon nanotubes are more and more often applied in ad-vanced polymer composites technology, but problem with aglomerization is still exist. Low-temperature plasma can be generated with a discharge between electrodes in a vacuum chamber. The process used to be carried out in the presence of gas. Depending on the medium applied, surface of modified material can be purified, chemically acti-vated, or grafted with various functional groups. Surface modification of powder can improve interac-tion between rubber matrix and filler (Mittal at al.;2011). In this work multi-walled carbon nanotubes (MWCNT) were modified with low-temperature plasma. Attempts were made to graft some functional groups on plasma-activated material to make filler's surface chemically active during processing. The analysis of surface free energy of virgin and modi-fied fillers was carried out. Ultimately, rubber com-posites were produced, and their mechanical proper-ties were studied.
\end{abstract}

Keywords - RF PECVD method, surface modifica-tion, fillers, rubber composites.

\section{INTRODUCTION}

In modern polymer industry there still is a room for new fillers, which can improve composites performance or introduce a new function to the material. New generation powder fillers like carbon nanotubes $(\mathrm{CNT})$ or graphene nanoplatelets $(\mathrm{GnP})$, are more and more often applied in advanced polymer composites technology. One of the most important issues is compatibility between polymer matrix and filler. Very often it is hard to obtain a well dispersed system when filler's particles are not wet properly by the polymer during mixing process. As a

\footnotetext{
${ }^{1}$ Institute of Polymers and Dyes Technology, Faculty of Chemistry, Technical University of Łódź, Stefanowskiego 12/16 Street, 90-924 Łódź, Poland.

${ }^{2}$ Institute of Materials Engineering, Faculty of Mechanical Engineering, Technical University of Łódź, Stefanowskiego 1/15 Street, 90-924 Łódź, Poland

${ }^{3}$ Institute of Electronic Materials Technology, Wólczyńska 133 Street, Warsaw, Poland
}

consequence, the total effect of mechanical properties improvement of a composite is lower than expected. We should avoid formation of big secondary filler structures to amplify the strengthening effect of filler, especially when advanced material like CNT GnP is used. It is possible to achieve by tailoring of material's surface properties - it should be wet by the polymer during processing and, if possible, take a part in crosslinking (in case of elastomer composites processing). Development of elastomer composites containing CNT seem to play bigger and bigger role due to acceptable prices of CNT, and extending applications of such composites, e.g. in modern tire industry.

Carbon materials in general are commonly used as fillers in elastomer industry. Their major role is to improve the mechanical properties of the vulcanizates. Too low surface free energy (SFE) of a filler can dramatically decrease the interaction between filler and the polymer matrix. In the other hand - too high SFE can intensify the agglomeration [1,2]. The possibility of controlling the dispersive part of carbon filler's surface free energy is strongly limited, so changes can only be achieved by tailoring it's polar part. in many cases applied matrix-filler compatibilization methods are not effective. Among last years some attempts were made to adapt low-temperature plasma to filler surface modification [3,4].

Carbon nanotubes, since their structures were discovered, focused interest of scientific groups from different sectors [5]. Due to its unique mechanical, electrical and thermal properties CNT's immidiately became one of most promising materials for advanced composites fillers [6]. Unfortunately it was soon found, that there is a major problem with achieving a satisfactory dispersion in polymer matrix.

One of often applied chemical methods for surface layer activation is solvent treatment, which generates of covalently bonded acidic, amine or fluorine groups [7]. The process is effective, but has also few major disadvantages - e.g. harmful reagent's environment, or generation of dangerous waste. More and more often attempts to modification with alternative methods, such as low-temperature plasma treatment, are observed. The applications of plasma techniques among polymer industry are very promising, especially due to environment protection issues - plasma treatment does not generate waste, is fast, and energetically advantageous process.

Low-temperature plasma can be observed as a discharge between electrodes in low-pressure reactor chamber. The discharge is maintained in a presence of a process gas, which for example can be Ar2, O2, H2, N2, acetylene, methane, or simply air. Depending on a type of gas used an controlling of process 
parameters, plasma treatment can be used for cleaning of the material's surface(so called "micro-sandblasting"), or etching of some functional groups via material's reaction with ionized gas particles. For different material and purposes a variety of generator frequencies can be applied - from $\mathrm{kHz}$ up to $\mathrm{MHz}$. The process can be repeated, and the effect depending on the material is stable for from few minutes up to months(e.g. plasma activated polypropylene can be further processed after several weeks). Most important advantages of plasma technique can be pointed as follows: no solvents have to be used, explosion protection is not necessary, staff is not exposed to toxic chemicals, modified materials do not have to be dried, running costs are very low, and last but not least - all types of solid materials can be treated with plasma.

Our previous studies revealed, that low-temperature plasma causes changes to surface free energy and its component of carbon nanotubes [7]. Plasma modification is a good method of CNT purification as an amorphous carbon is eliminated from their surface during process [8].

In this work multi-walled carbon nanotubes (MWCNT) were modified with low-temperature plasma. Attempts were made to graft some functional groups on plasma-activated material to make filler's surface chemically active during processing and more compatible with polymer matrix. The analysis of surface free energy of virgin and modified fillers was carried out. Ultimately rubber composites were produced, and their mechanical properties were studied.

\section{EXPERIMENTAL}

\section{MATERIALS}

\section{A. Multiwalled carbon nanotubes}

Two types of powder fillers were the objects of the study: $\mathrm{COOH}$ functionalized and $\mathrm{OH}$ funcionalized carbon multiwalled nanotubes (Cheap Tubes Inc, USA). CNT purity was $>98 \mathrm{wt} \%$.

\section{B. Silanes}

Silane M - silane containing mercaptane chemical groups(Sigma Aldrich, Germany)

Silane V - silane containing vinyl chemical groups(Sigma Aldrich, Germany)

\section{Rubber}

The rubber used in the study was styrene-butadiene rubber SBR KER 1500(Synthos, Polska).

\section{TECHNIQUES}

\section{A. Plasma assisted modification of MWCNT}

Fillers studied were modified with a Zepto tumbler plasma reactor (Diener, Germany). The reactor operated with the frequency of $40 \mathrm{kHz}$ and the discharge power of 30, 60 and 100 W. Scheme of the reactor is shown in Fig. 1.

As a first stage, MWCNT activation(in power of 30, 60 and $100 \mathrm{~W}$ ) was carried out in oxygen plasma in time of 4 to $32 \mathrm{~min}$. with oxygen flow at the level of $30 \mathrm{sccm}$. Right after activation, silane vapours were introduced into reactor chamber and the flow at the level of $20 \mathrm{sccm}$ was maintained in a time of $10 \mathrm{~min}$.

\section{B. Surface free energy of fillers}

Effectiveness of fillers modification is represented by changes of surface free energy (SFE) and its components - polar and dispersive. SFE was examined with a K100 MKII tensiometer (KRÜSS GmbH, Germany). Contact angle was determined using polar (water, methanol, ethanol) and non-polar (n-hexane, n-heptane) liquids. SFE and its components were calculated by the method proposed by Owens-Wendt-Rabel-Kaeble [5].

\section{Rubber mixes production}

Rubber mixes were prepared with a Brabender Plasticorder laboratory micro mixer (Germany), operated with $45 \mathrm{rpm}$, during $30 \mathrm{~min}$. Their composition is presented in Table 1. The only one variable was the type of modified mineral filler. Sampes were vulcanized in $160^{\circ} \mathrm{C}$. Mixes components were as follows: rubber(100 phr), $\mathrm{ZnO}$ (3,5 phr), stearic acid(1 phr), CBS (2phr), sulphur (2 phr).

\section{Mechanical properties of rubber vulcanizates}

Mechanical properties of the vulcanizates were determined with a Zwick 1435 universal mechanical testing machine (Germany). Tests were carried out on "dumbbell" shape, 1.5 mm thick and $4 \mathrm{~mm}$ width specimens, according to PN-ISO 37:1998 standard. The following properties of the materials were determined: elongation at break $(\mathrm{Eb})$, stress at elongation of $100 \%$ (SE100), 200\% (SE200), 300\% (SE300) and tensile strength (TS).

\section{E. Crosslinkig density of rubber vulcanizates}

Crosslinking density was determined with use of Flory's formula [6]:

$$
v=-\frac{1}{V_{0}} \cdot \frac{\ln \left(1-V_{r}\right)+V_{r}+\mu V_{r}^{2}}{\sqrt[3]{V_{r}}-\frac{2 V_{r}}{f}}
$$

Where:

$v$ - crosslinking density $\left[\mathrm{mol} / \mathrm{cm}^{3}\right]$;

$V_{0}$ - volume of solvent in swollen sample;

$V_{r}$-volume of polymer in swollen sample;

$f$-functionality of crosslinking $(f=4)$;

$\mu$-Huggins parameter;

As a solvent toluene were used. Samples were weighed before swollen, after swollen and in the third step after drying. 2.6. Scanning electron microscopy (SEM)

Surface of CNT samples was studied with an AURIGA (Zeiss, Germany) scanning electron microscope (SEM). Secondary electron signal (SE) was used for surface imaging. Accelerating voltage of the electron beam was set to $10 \mathrm{keV}$.

\section{RESULTS AND DISCUSSION}

\section{A. Surface free energy (SFE)}

Surface free energy and its components for CNT-COOH modified with Silane M did not changed significantly(Tab. 2) - 
a slight decrese of the polar part was observed in case of $100 \mathrm{~W}$ treatment. Grafting of Silane V in $60 \mathrm{~W}$ discharge power leads to the decrease of polar part of surface free energy -it means that polar, oxygene containing groups were substituted with a silane. Perhaps in this case $30 \mathrm{~W}$ of discharge power is not enough to initiate grafting, and in the other hand $100 \mathrm{~W}$ provide to destruction of silane particles.

In case of CNT-OH only treatment in $30 \mathrm{~W}$ leads to decrease of surface free - for both silanes(Tab. 3).

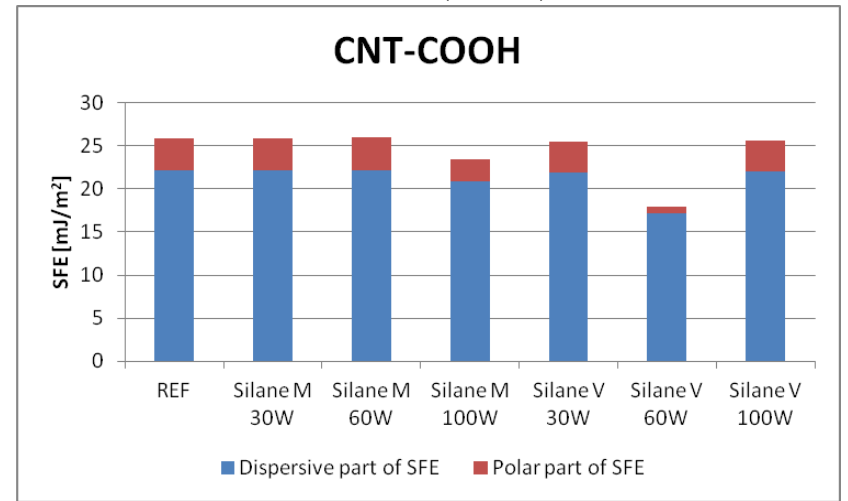

Fig. 1. Surface free energy of CNT-COOH samples

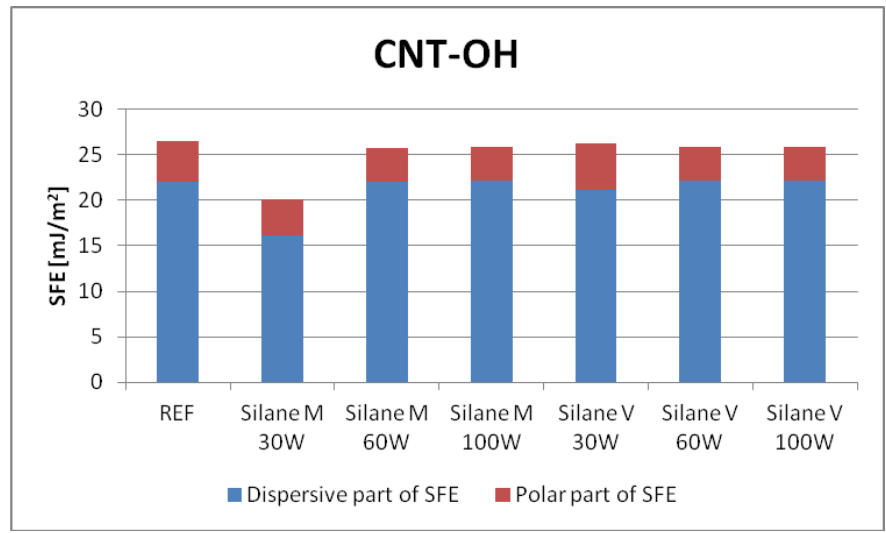

Fig. 2. Surface free energy of CNT-OH samples

\section{B. Mechanical properties of rubber vulcanizates containing modified CNT}

Mechanical properties of composites usually changes if we affect the interaction between polymer and filler - modules at 100, 200 and 300\% elongation should be bigger for better interaction. Material performance at small elongations is often underestimated - it is very important as vulcanizates usually „,work” at rather small deformations.

In case of Silane M modified CNT-COOH we can observe the increse of mechanical modulus for 60 and $100 \mathrm{~W}$ discharge treatment. Similar, however lower scale effect is observed for Silane V modified samples. One have to keep in mind that during treatment in plasma reactor CNT are exposed to conditions which leads also to material degradation in higher discharge power. This phenomena will not be reflected in SFE results, but will affect mechanical performance of composites. As a consequence in mechanical properties results two effects can overlap - CNT chemical modification and change in the geometry (eg. smaller CNT length that can have strengthening effect on elastomer).
In case of CNT-OH treated with Silane $\mathrm{M}$ strengthening effect is observed for 30 and $60 \mathrm{~W}$ treatment what stays in acordance to SFE results. Silane V treatment doesn't affect mechanical performance.

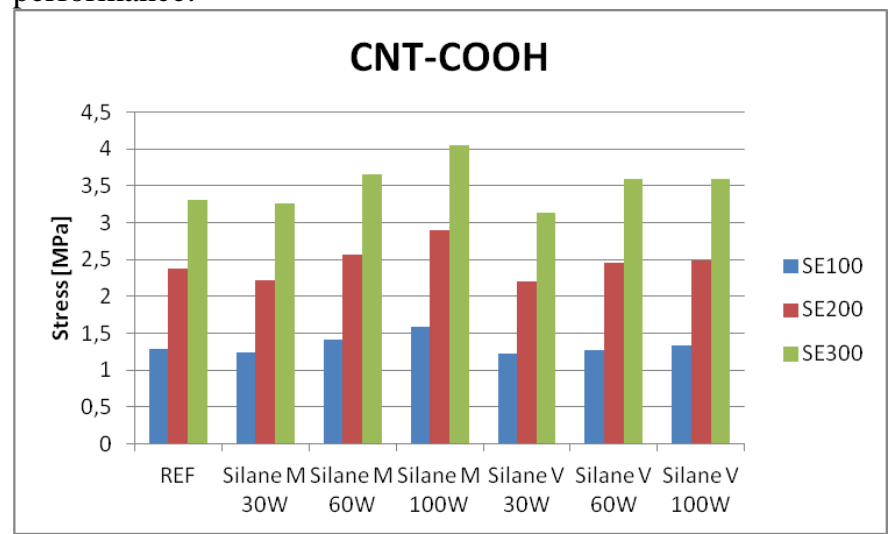

Fig. 3. Mechanical properties of rubber vulcanizates filled with CNT-COOH; SE - stress at elongation 100, 200,300\%

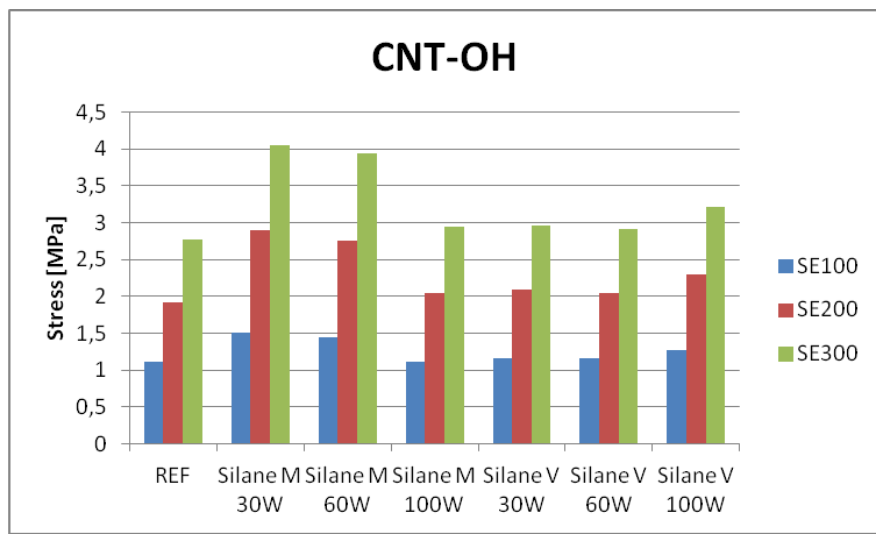

Fig. 4. Mechanical properties of rubber vulcanizates filled with CNT-OH; SE stress at elongation 100, 200,300\%

\section{Crosslinking density of rubber vulcanizates}

Proposed surface modification of CNT is expected to lead to increased material's activity during crosslinking process. In cace of CNT-COOH any significant changes were observed. It leads to the conclusion, that for better mechanical performance of this composites probably responsible is phenomena of CNT degradation and length reduction in plasma atmosphere.

Regarding to CNT-OH we observed an interesting results for effectively modified samples(Silane M 30 and 60W) the crosslink density was approximately $20 \%$ higher than for a refference sample, and it is reflected by mechanical performance and SFE results. 


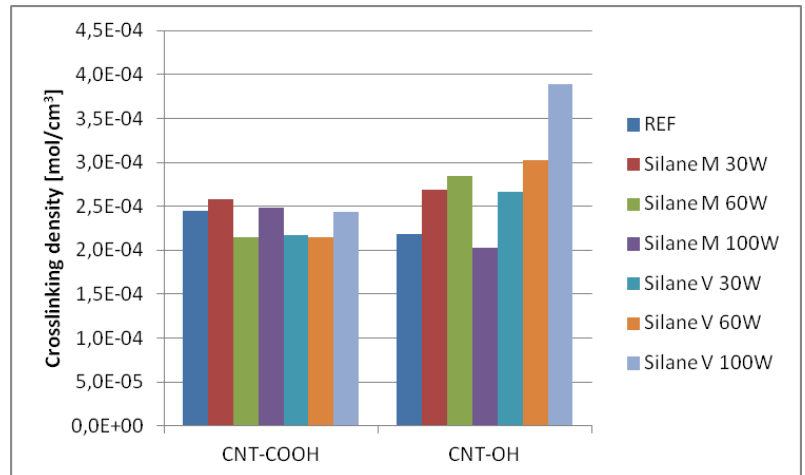

Fig. 5. Crosslinking density of rubber vulcanizates filled with $\mathrm{CNT}-\mathrm{COOH}$ and CNT-OH

\section{Scanning electron microscopy (SEM)}

To investigate the differences between samples SEM - ESB technique was applied. In ESB mode one can observe a mass contrast on samples surface. It occurred, that depending on the CNT and silane type materials after modification differs significantly. Bright regions on the images are attributed to silica atoms, which reflects the presence of silane particles on CNT surface. It was found that much higher amount of silane was transferred to the samples in case of CNT-OH treatment, and the process was more effective for Silane M.
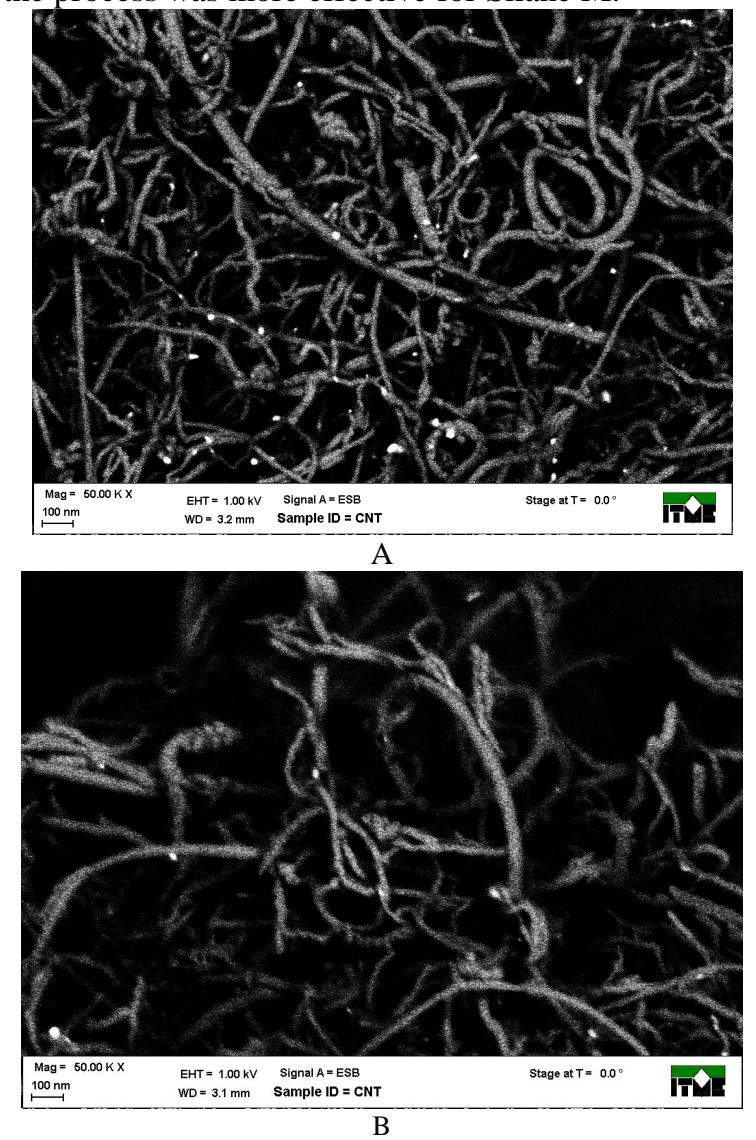

Fig. 6. Scanning electron microscopy of :(A) CNT-OH treated with Silane M in 30W plasma, (B) CNT-COOH treated with Silane V in 60W plasma.

\section{CONCLUSION}

1. Significant reduction of SFE polar part was achieved for CNT-COOH treated with Silane V in 60W discharge power, and for CNT-OH treated with Silane M in $30 \mathrm{~W}$.

2. An increse of mechanical modulus was observed for composites containing CNT-COOH modified with Silane $\mathrm{M}$ in 60 and 100W and CNT-OH in Silane M in 30 and 60 W.

3. Significant cross ling density increse was observed in case of vulcanizates containing Silane M modified CNT-OH.

4. Probably in case of CNT-OH chemical grafting was more effective, while for CNT-COOH the results are overlapped by the phenomena of material degradation during plasma treatment.

\section{ACKNOWLEDGMENT}

The project was funded by the National Science Centre Poland $(\mathrm{NCN})$ conferred on the basis of the decision number DEC-2012/05/B/ST8/02922.

\section{REFERENCES}

[1] Wolff S.; Wang J. Filler-Elastomer Interactions. Part IV. The Effect of the Surface Energies of Fillers on Elastomer Reinforcement. Rubber Chemistry and Technology 1992, 65, 329-342.

http://dx.doi.org/10.5254/1.3538615

[2] Dierkes W. K.; Guo R.; Mathew T.; Tiwari M.; Datta R. N.; Talma A. G.; Noordemeer J. W. M.; van Ooij W. J. A key to enhancement of compatibility and dispersion in elastomer blends. Kautschuk Gummi Kunststoffe 2011, 64, 28-35.

[3] Chityala A.; van Ooij W. J. Plasma deposition of polymer films on pmma powders using vacuum fluidisation techniques. Surface Engineering 2000, 16, 299-302.

http://dx.doi.org/10.1179/026708400101517279

[4] Bieliński D.; Parys G.; Szymanowski H. Plazmochemiczna modyfikacja powierzchni sadzy jako napełniacza mieszanek gumowych. Przemysł Chemiczny 2012, 91, $1508-1512$.

[5] Owens D. K.; Wendt R. C. Estimation of the surface free energy of polymers. Journal of Applied Polymer Science 1969, 13, 1741-1747. http://dx.doi.org/10.1002/app.1969.070130815

[6] H. Janik, A. Balas, Budowa chemiczna i właściwości fizyczne usieciowanych poliuretanów segmentowych, Polimery 2009, 3, 195

[7] Siciński M., Bieliński D., Gozdek T., Piątkowska A., Kleczewska J., Kwiatos K., Kompozyty elastomerowe z dodatkiem grafenu lub MWCNT modyfikowanych plazmochemicznie. Inżynieria Materiałowa 2013, 6,

[8] Xu T.; Yang J.; Liu J.; Fu Q. Surface modification of multi-walled carbon nanotubes by O2 plasma. Applied Surface Science 2007, 253, 8945-8951.

http://dx.doi.org/10.1016/j.apsusc.2007.05.028 